\title{
Ship waves on a viscous fluid of finite depth
}

\author{
Andy T. Chan and Allen T. Chwang \\ Department of Mechanical Engineering, The University of Hong Kong, Pokfulam Road, Hong Kong
}

(Received 8 July 1996; accepted 10 December 1996)

\begin{abstract}
In this paper we are concerned with the wave generation by a singular forcelet in a viscous fluid of finite depth, where the singularity is located far from the bottom and not very near the free surface. In the first part of this work, the image system of an Oseenlet bounded by a no-slip wall, is considered. It is found that the resultant velocity field can be described by a planar distribution of vertical Oseen doublets and a negative Oseenlet located at the mirror point of the singularity with respect to the plane wall. In the second part of the work we deal with the generation of waves by these solutions. By imposing the linearized free-surface conditions on the solutions obtained from the first part, the wave generated is shown to exhibit the Kelvin ship wave pattern that agrees with observation. The effects of water depth and of submergence on the wave amplitude are also investigated. (C) 1997 American Institute of Physics. [S1070-6631(97)02504-X]
\end{abstract}

\section{INTRODUCTION}

When a submerged body cruises with constant velocity in an inviscid fluid, it induces on the free surface the characteristic Kelvin's ship wave pattern. Several methods for determining the wave generated by a moving source in an inviscid fluid are available (see Wehausen and Laitone ${ }^{1}$ ). Cumberbatch ${ }^{2}$ studied viscous ship waves for a pressure disturbance. Dugan ${ }^{3}$ studied the two-dimensional version of the problem by considering the viscous drag of bodies moving near a free surface. Wilson ${ }^{4}$ developed a linear theory for the free surface flow of a viscous fluid past point mass sources and forcelets. Chan and Chwang ${ }^{5}$ have solved for the problem of ship wave generation in a viscous fluid of infinite depth. In this paper, we investigate the waves generated by a singular force (an Oseenlet) moving with a uniform velocity in a fluid of finite depth, which creates a laminar far wake downstream of itself.

As discussed by Chan and Chwang, ${ }^{5}$ the solution for the case of infinite depth has been successfully obtained by use of Fourier's transform. The solutions, which satisfy the linearized free-surface conditions, possess many characteristics of Kelvin's ship wave pattern, except that they also include a viscous decay term. These solutions will be used as the building blocks for the present study. The major problem, thus, lies in finding an "image system" to remove the noslip boundary. In solving the problem, two boundary conditions must be satisfied: one on the free surface and the other on the horizontal plane. The free surface can be treated in its linearized form, with linearized solutions serving as the basis. The no-slip boundary condition on the horizontal plane, however, is less frequently dealt with for an Oseenlet.

In the first part of this paper, we shall derive the image system of an Oseenlet bounded by a no-slip boundary. In the second part we will extend the first part of the work to derive the solutions for the wave generated by an Oseenlet.

\section{THE IMAGE SYSTEM OF AN OSEENLET NEAR A NO-SLIP BOUNDARY}

We first consider the problem of a steady threedimensional Oseen flow past a horizontal no-slip boundary, with the presence of a singular force acting at the opposite direction to the flow. The fundamental singular solution for a point force, known as the Lagerstrom needle, has been known for many years. We want to remove the physical boundary by finding the complementary terms to the Oseenlet situated at a distance from a stationary plane boundary, such that the no-slip boundary condition can be satisfied on the boundary.

Let us consider an unbounded viscous fluid moving with a constant velocity $U$ along the positive $x$ direction. The appropriate nondimensional Oseen equations to be satisfied in the domain are

$$
\begin{aligned}
& \boldsymbol{\nabla} \cdot \mathbf{u}=0, \\
& \frac{\partial \mathbf{u}}{\partial x}=-\nabla p+\epsilon \nabla^{2} \mathbf{u},
\end{aligned}
$$

where

$$
\epsilon=\frac{\mu g}{\rho U^{3}},
$$

$\rho$ and $\mu$ are the density and dynamic viscosity of the fluid, respectively. In Eqs. (1) and (2), the dimensionless pressure $p$ is nondimensionalized by $\rho U^{2}$, the velocity vector $\mathbf{u}$ by $U$, and distance by $U^{2} / g$, where $g$ is the gravitational constant. The dimensionless parameter $\epsilon$ can be regarded as the reciprocal of the Reynolds number with respect to the deep-water wavelength $U^{2} / g$. It will be used as the perturbation parameter throughout the asymptotic expansions in this paper.

For a singular force located at $(0,0, h)$ with magnitude $4 \pi F$, normalized with respect to $\rho U^{2}\left(U^{2} / g\right)^{2}$, along the negative $x$ direction, the solution to (1) and (2) for an unbounded fluid is given by Moore ${ }^{6}$ as

$$
\begin{aligned}
& \mathbf{u}_{u}[x, y, z-h]=-\frac{F}{\epsilon} \frac{e^{(x-r) / 2 \epsilon}}{r} \mathbf{e}_{\mathbf{x}}+F \boldsymbol{\nabla}\left(\frac{e^{(x-r) / 2 \epsilon}-1}{r}\right), \\
& p_{u}[x, y, z-h]=-\frac{F x}{r^{3}},
\end{aligned}
$$

where 


$$
r=\sqrt{x^{2}+y^{2}+(z-h)^{2}}
$$

and $\mathbf{e}_{\mathbf{x}}$ is the unit vector in the $x$ direction.

If the fluid only occupies the upper half-space $(z>0)$ and is bounded below by an infinite plate at $z=0$, the no-slip boundary condition must be imposed on the horizontal plane boundary. Similar to Blake, ${ }^{7}$ we locate an Oseenlet of equal magnitude but opposite in sign at the mirror image point $(0,0,-h)$ with respect to the plane wall. Therefore the resultant velocity field can be written as

$$
\begin{aligned}
& \mathbf{u}=\mathbf{u}_{u}[x, y, z-h]-\mathbf{u}_{u}[x, y, z+h]+\mathbf{u}_{b}, \\
& p=p_{u}[x, y, z-h]-p_{u}[x, y, z+h]+p_{b},
\end{aligned}
$$

where $\mathbf{u}_{b}$ and $p_{b}$ are the unknown functions introduced to satisfy the no-slip boundary conditions. In order to make the tangential component of the total velocity $\mathbf{u}$ given by (7) vanish on the plane boundary, the tangential component of $\mathbf{u}_{b}$ must also vanish on the plane boundary. Hence the problem is much simplified in the sense that the boundary conditions are simpler to deal with.

The velocity $\mathbf{u}_{b}$ at the plane boundary can be expressed in terms of the Fourier integral as

$$
\begin{aligned}
\left.\mathbf{u}_{b}\right|_{z=0}= & \frac{F}{2 \pi} \int_{-\infty}^{\infty} \int_{-\infty}^{\infty} e^{i\left(k_{1} x+k_{2} y\right)} \\
& \times \frac{2\left(e^{-B h}-e^{-A h}\right)}{A B} d k_{1} d k_{2} \mathbf{e}_{z},
\end{aligned}
$$

where

$$
A=\sqrt{k_{1}^{2}+k_{2}^{2}} \quad \text { and } \quad B=\sqrt{k_{1}^{2}+k_{2}^{2}+\frac{i k_{1}}{\epsilon}} .
$$

It follows from Olmstead ${ }^{8}$ that a solution of the Oseen equations (1) and (2) that satisfies the boundary conditions can be given by a distribution of vertical Oseen doublets or its higher derivatives in the form of

$\mathbf{u}_{b}=-\int_{-\infty}^{\infty} \int_{-\infty}^{\infty} \frac{\partial \mathbf{u}_{u}}{\partial z}\left[x-x^{\prime}, y-y^{\prime}, z\right] N\left[x^{\prime}, y^{\prime}\right] d x^{\prime} d y^{\prime}$,

where $N[x, y]$ is the distribution function of Oseen doublets on the plane wall.

By applying the boundary conditions to (11) and applying the convolution theorem, we obtain the Fourier transform of $N[x, y]$ as

$$
\hat{N}\left[k_{1}, k_{2}\right]=\frac{2\left(e^{-B h}-e^{-A h}\right)}{B-A},
$$

where $\hat{N}\left[k_{1}, k_{2}\right]$ is the Fourier transform of $N[x, y]$.

By writing $k_{1}=i \xi$, inverse Fourier transform of $\hat{N}$ with respect to $x$, denoted by $I_{1}$, can be shown to be

$$
I_{1}=-\frac{1}{2 \pi} \int_{-\infty}^{\infty} e^{i k_{2} y} \int_{-\infty}^{\infty} 2 i e^{-x \xi} \frac{e^{-h B^{\prime}}-e^{-h A^{\prime}}}{B^{\prime}-A^{\prime}} d \xi d k_{2},
$$

where

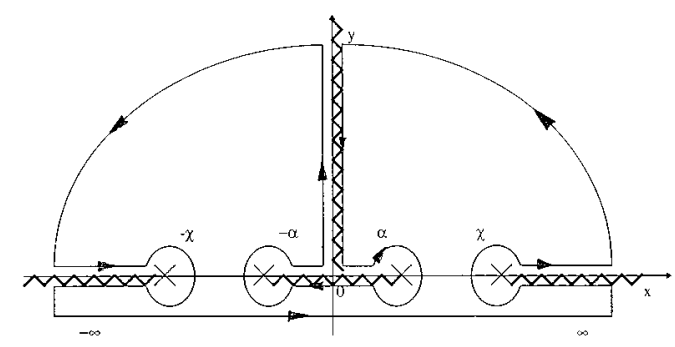

FIG. 1. Contour used in evaluating the $k_{2}$ integral.

$$
B^{\prime}=\sqrt{k_{2}^{2}-\left(\xi^{2}+\frac{\xi}{\epsilon}\right)}=\sqrt{k_{2}^{2}-\alpha^{2}}
$$

and

$$
A^{\prime}=\sqrt{k_{2}^{2}-\xi^{2}}
$$

The $k_{2}$ integral is more complicated, due to the presence of branch cuts at $A^{\prime}$ and $B^{\prime}$, respectively. However, the evaluation can be simplified by considering the contour as in Fig. 1. After performing the contour integrations, we obtain the distribution function $N[x, y]$ as

$$
\begin{aligned}
N[x, y]= & \frac{8}{\pi} \int_{0}^{\infty} \int_{0}^{\xi} e^{-x \xi} \cos y \zeta \\
& \times \frac{\cos \left[h \sqrt{\alpha^{2}-\zeta^{2}}\right]-\cos \left[h \sqrt{\xi^{2}-\zeta^{2}}\right]}{\sqrt{\alpha^{2}-\zeta^{2}}-\sqrt{\xi^{2}-\zeta^{2}}} d \xi d \zeta .
\end{aligned}
$$

With the substitutions

$$
\zeta=\xi \cos \lambda \quad \text { and } \quad \xi=\frac{\cos \lambda}{\epsilon \sin ^{2} \lambda \cos \vartheta},
$$

Eq. (16) can be expressed in terms of trigonometric functions as

$$
\begin{aligned}
N[x, y]= & \frac{32 \epsilon}{\pi} \int_{0}^{\pi / 2} \int_{0}^{\pi / 2} e^{-x C} \cos [y C \cos \lambda \tan \vartheta] \\
& \times \frac{\cos [h C]-\cos [h C(\cos \lambda / \cos \vartheta)]}{\cos \vartheta-\cos \lambda} \\
& \times \cot \lambda d \lambda d \vartheta
\end{aligned}
$$

where

$$
C=\frac{\cos \lambda}{\epsilon \sin ^{2} \lambda \cos \vartheta}
$$

Equations (18) and (19) cannot be further integrated to give exact solutions. However, Eq. (18), despite its complicated form, does give some important information about the distribution of Oseen doublets on the plane. First of all, it can be shown that (18) is convergent, whereas in (16) this is not explicit. From (18) it also shows that the distribution function $N[x, y]$ is of the order of $\epsilon$, which has important consequences on two aspects. Physically, as $\epsilon$ decreases to zero, the two Oseenlets actually become a three-dimensional inviscid source-like singularity, and the distribution function vanishes. This agrees with the simple mirror image theory for a 


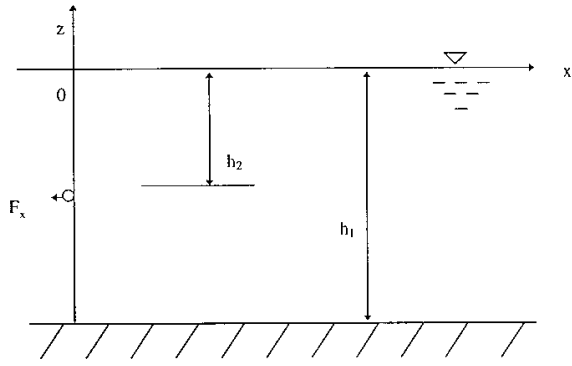

FIG. 2. The coordinate system for an Oseenlet in a bounded fluid.

plane wall in potential flow theory. Second, it will be shown later that this distribution of singularities has little effect on the generation of surface waves for large Reynolds numbers in the wave amplitude evaluation. This is because, as shown by Chan and Chwang, ${ }^{5}$ the major contribution to surface waves generated by moving submerged bodies is due to the inviscid terms, which are of the order of $O[1]$, while the contribution due to the viscous terms is of the order of $\sqrt{ } \epsilon$.

The distribution function $N[x, y]$ also decays exponentially along the positive $x$ direction, and the upstream of the singularity has no effect on the flow field. This is very similar to the two-dimensional case derived by Olmstead and Gautesen. ${ }^{9}$ Although the distribution function is now much more complex than in the two-dimensional case, this characteristic, which originates because of the asymmetry of the Oseen flow field, is still retained.

With immediate reference to Blake, ${ }^{7}$ it is clear that the Oseenlet image system is more complicated than that of the Stokeslet because of the inclusion of the inertial effect. The Stokeslet image system is simply the sum of the Stokes doublet, a negative Stokeslet, and a source doublet. Another point worth mentioning is that the presence of the no-slip boundary does not allow simple superposition. This has been illustrated for the Stokeslet case by Blake and Chwang. ${ }^{10}$ The image system, for higher derivatives of the Oseenlet, which requires extensive work, is expected to be much more complex.

\section{SURFACE WAVE GENERATION BY AN OSEENLET WITH A HORIZONTAL NO-SLIP BOUNDARY}

In the previous section, we have removed the no-slip boundary by introducing the complementary terms to the Oseen equations. This means that the situation has changed from an Oseenlet with a no-slip boundary to two Oseenlets of different signs plus a distribution of vertical Oseen doublets midway between them in an unbounded fluid.

Chan and Chwang ${ }^{5}$ have considered the problem of a submerged Oseenlet in infinitely deep water with a free surface. The present problem is actually similar to that considered by Chan and Chwang, ${ }^{5}$ but with two separate Oseenlets and a planar distribution of Oseen doublets. We now consider the general problem of finding the wave amplitude generated by an Oseenlet submerged at a depth $h_{2}$ from the free surface in a viscous fluid of depth $h_{1}$ as in Fig. 2. We assume that $h_{1}-h_{2}$ is large enough so that at distances downstream of the singularity, the wake region does not interact much with the boundary layer on the no-slip boundary, so that the Oseen equations are still valid. The submergence depth $h_{2}$ should not be too small also, because of the assumption of weak disturbance at the free surface in the use of linearized free surface boundary conditions and the governing Oseen equations.

As mentioned earlier, the no-slip boundary can be removed by locating an Oseenlet of opposite sign at $\left(0,0,-2 h_{1}+h_{2}\right)$ and a planar distribution $N[x, y]$ of vertical Oseen doublets at $z=-h_{1}$. We now impose the free-surface conditions at $z=0$. The linearized free-surface conditions represent the vanishing of normal and shear stresses at $z=0$,

$$
\begin{aligned}
& \frac{\partial u}{\partial z}+\frac{\partial w}{\partial x}=0, \\
& \frac{\partial v}{\partial z}+\frac{\partial w}{\partial y}=0, \\
& \frac{\partial p}{\partial x}-2 \epsilon \frac{\partial^{2} w}{\partial x \partial z}-w=0 .
\end{aligned}
$$

The procedure for deriving the wave amplitude $\eta$ is similar to that of a single Oseenlet as in Chan and Chwang, ${ }^{5}$ which will not be reproduced. However, we shall simply quote the exact solution for the wave amplitude generated by a single Oseenlet located at depth $z_{0}$ from the free surface:

$$
\eta=\eta_{u}+\eta_{\mathrm{FS}}, \quad \eta_{\mathrm{FS}}=\eta_{\mathrm{FS}}^{o}+\eta_{\mathrm{FS}}^{v} .
$$

where the subscript FS denotes the free-surface effect and the superscripts $o$ and $v$ denote the contribution from the inviscid terms $\nabla[1 / r]$ and viscous terms of the Lagerström needle, respectively, with

$$
\begin{gathered}
\eta_{\mathrm{FS}}^{o}\left[z_{0}\right]=F \int_{-\infty}^{\infty} \int_{-\infty}^{\infty} e^{i\left(k_{1} x+k_{2} y\right)}\left[\left(i k_{1}+2 \epsilon A^{2}\right) \hat{\Phi}_{0}\right. \\
\left.\quad+2 i \epsilon k_{2} B \hat{\Psi}_{0}\right] d k_{1} d k_{2}, \\
\eta_{\mathrm{FS}}^{v}\left[z_{0}\right]=F \int_{-\infty}^{\infty} \int_{-\infty}^{\infty} e^{i\left(k_{1} x+k_{2} y\right)}\left[\left(i k_{1}+2 \epsilon A^{2}\right) \hat{\Phi}_{v}\right. \\
\left.\quad+2 i \epsilon k_{2} B \hat{\Psi}_{v}\right] d k_{1} d k_{2}, \\
\hat{\Phi}_{0}=\frac{\left[-\left(k_{1}^{2} / 2 A+\frac{1}{2}\right)+2 i \epsilon k_{1} A+2 \epsilon^{2} A^{2}(A+B)\right] e^{-A z_{0}}}{\Delta}, \\
\hat{\Phi}_{v}=\frac{\left(-2 i \epsilon k_{1} B-4 \epsilon^{2} A^{3} B\right) e^{-B z_{0}}}{\Delta}, \\
\hat{\Psi}_{0}=\frac{\left(-2 \epsilon k_{1} A+4 i \epsilon^{2} A^{4}\right) e^{-A z_{0}}}{\Delta}, \\
\hat{\Psi}_{v} \frac{\left[(i / 2)\left(k_{1}^{2}-A\right)+2 \epsilon k_{1} A^{2}-2 i \epsilon^{2} A^{4}-2 i \epsilon^{2} A^{3} B\right] e^{-B z_{0}}}{\Delta},
\end{gathered}
$$

and the pole equation $\Delta$ given by

$$
\Delta=\left(k_{1}^{2}-A\right)-4 i \epsilon k_{1} A^{2}+4 \epsilon A^{3}(B-A) .
$$


The asymptotic expressions for the wave amplitude generated by an Oseenlet with magnitude $4 \pi F$ at a depth of submergence $z_{0}$ for small values of $\epsilon$ have been shown by Chan and Chwang ${ }^{5}$ as

$$
\eta \approx \eta_{\mathrm{FS}}=\eta_{o}+\eta_{v}+O[\epsilon],
$$

where $\eta_{o}$ and $\eta_{v}$ represent the wave amplitude contribution from the inviscid part and the remaining viscous part of (4), respectively. In a polar coordinate system $(R, \theta)$ on the undisturbed free surface with

$$
x=R \cos \theta \text { and } y=R \sin \theta,
$$

the expressions for $\eta_{o}$ and $\eta_{v}$ are given by

$$
\begin{aligned}
\eta_{o}\left[z_{0}\right]= & 4 F\left(\frac{2 \pi}{R \cos \theta}\right)^{1 / 2}\left(1-8 \tan ^{2} \theta\right)^{-1 / 4}\left(( \frac { m ^ { + } + 1 } { 2 } ) ^ { 3 / 4 } e ^ { - ( z _ { 0 } / 2 ) ( 1 + m ^ { + } ) - ( 4 \epsilon R / m ^ { + } ) [ ( m ^ { + } + 1 ) / 2 ] ^ { 3 } \operatorname { c o s } \theta } \operatorname { c o s } \left\{R\left(\frac{m^{+}+1}{2}\right)^{1 / 2}\right.\right. \\
& \left.\times\left[\cos \theta-\left(\frac{m^{-}-1}{2}\right)^{1 / 2} \sin \theta\right]-\frac{\pi}{4}\right\}+\left(\frac{m^{-}+1}{2}\right)^{3 / 4} e^{-\left(z_{0} / 2\right)\left(1+m^{-}\right)-\left(4 \epsilon R / m^{-}\right)\left[\left(m^{-}+1\right) / 2\right]^{3} \cos \theta} \cos \left\{R\left(\frac{m^{-}+1}{2}\right)^{1 / 2}\right. \\
& \left.\left.\times\left[\cos \theta-\left(\frac{m^{+}-1}{2}\right)^{1 / 2} \sin \theta\right]+\frac{\pi}{4}\right\}\right), \\
\eta_{v}\left[z_{0}\right]= & 4 \sqrt{2 \epsilon} F\left(\frac{2 \pi}{R \cos \theta}\right)^{1 / 2}\left(1-8 \tan ^{2} \theta\right)^{-1 / 4}\left(\frac{m^{+}+1}{2}\right)^{3 / 2} e^{-\left(z_{0} / \sqrt{2 \epsilon}\right)\left(1+m^{+}\right)^{1 / 4}-\left(4 \epsilon R / m^{+}\right)\left[\left(m^{+}+1\right) / 2\right]^{3} \cos \theta} \\
& \times \cos \left\{R\left(\frac{m^{+}+1}{2}\right)^{1 / 2}\left[\cos \theta-\left(\frac{m^{+}-1}{2}\right)^{1 / 2} \sin \theta\right]-\frac{z_{0}}{\left.\sqrt{2 \epsilon}\left(1+m^{+}\right)^{1 / 4}+\frac{\pi}{2}\right\}}\right. \\
& +\left(\frac{m^{-}+1}{2}\right)^{3 / 2} e^{-\left(z_{0} / \sqrt{2 \epsilon}\right)\left(1+m^{-}\right)^{1 / 4}\left(4 \epsilon R / m^{-}\right)\left[\left(m^{-}+1\right) / 2\right]^{3} \cos \theta} \cos \left\{R ( \frac { m ^ { - } + 1 } { 2 } ) ^ { 1 / 2 } \left[\cos \theta-\left(\frac{m^{-}-1}{2}\right)^{1 / 2}\right.\right. \\
& \times \sin \theta]-\frac{z_{0}}{\left.\left.\sqrt{2 \epsilon}\left(1+m^{-}\right)^{1 / 4}+\pi\right\}\right)}
\end{aligned}
$$

and

$$
m^{ \pm}=\frac{\cot ^{2} \theta}{4}\left(1 \pm \sqrt{1-8 \tan ^{2} \theta}\right) .
$$

Therefore the resultant wave amplitude for the no-slip boundary system can be expressed as

$$
\begin{aligned}
\eta_{0}^{B}= & \eta_{0}\left[h_{2}\right]-\eta_{0}\left[2 h_{1}-h_{2}\right]+\int_{-\infty}^{\infty} \int_{-\infty}^{\infty} \eta_{0}\left[x-x^{\prime}, y\right. \\
& \left.-y^{\prime}, h_{1}\right] N\left[x^{\prime}, y^{\prime}\right] d x^{\prime} d y^{\prime}, \\
\eta_{v}^{B}= & \eta_{v}\left[h_{2}\right]-\eta_{v}\left[2 h_{1}-h_{2}\right]+\int_{-\infty}^{\infty} \int_{-\infty}^{\infty} \eta_{v}\left[x-x^{\prime}, y\right. \\
& \left.-y^{\prime}, h_{1}\right] N\left[x^{\prime}, y^{\prime}\right] d x^{\prime} d y^{\prime} .
\end{aligned}
$$

We now look at the relative order of the respective terms for small values of $\epsilon$. It has been shown by Chan and Chwang ${ }^{5}$ and Cumberbatch ${ }^{2}$ that the asymptotic behavior of the viscous wave amplitude $\eta_{v}$ is of the order of $\sqrt{ } \epsilon$. At the same time, the distribution is of the order of $\epsilon \exp [-x / \epsilon]$, which diminishes rapidly for small $\epsilon$ and large distances from the singularity. The relative order of the $N[x, y]$ distribution terms also decreases with increasing submergence as the wave amplitude $\eta_{0}$ and $\eta_{v}$ decrease with the order of $\exp \left[-z_{0} / \epsilon\right]$.
Therefore, the dominant terms in the wave amplitude for small values of $\epsilon$ are still the inviscid part of the Oseenlet solution plus its mirror image solution, which is of the same order as the Oseenlet, except for the different submergence. Therefore all the properties of the viscous Kelvin's wave pattern described by Chan and Chwang ${ }^{5}$ are expected to retain, except that the wave amplitude is significantly reduced for smaller depth $h_{1}$. The free-surface profile for an Oseenlet with magnitude $F=\frac{1}{4}$ at $\epsilon=0.001, h_{1}=10$, and $h_{2}=1$ are shown in Fig. 3.

An interesting point of study is to investigate the effect of water depth $h_{1}$ on the wave amplitude. The maximum wave amplitude along the line of motion is plotted in Fig. 4 as a surface against the normalized depth $h_{1}$ at $\epsilon=0.001$ and $h_{2}=1$. An immediate conclusion drawn from the plots is that the wave amplitude increases with increasing water depth $h_{1}$. Far away from the Oseenlet, the effect of the no-slip boundary is so small that the wave amplitude asymptotically reaches the value for the infinite water depth case, as in Chan and Chwang. ${ }^{5}$ Physically the no-slip boundary acts to reduce the velocity and generates vorticity at its near field. Therefore for small $h_{1}$, the effect of the no-slip boundary tends to reduce the overall velocity of the system, and thus a smaller wave is expected to be generated. In fact, the wave amplitude is only significantly reduced when $h_{2}=O\left[h_{1}\right]$ : that is, either the water depth is very small, or the Oseenlet is very close to 


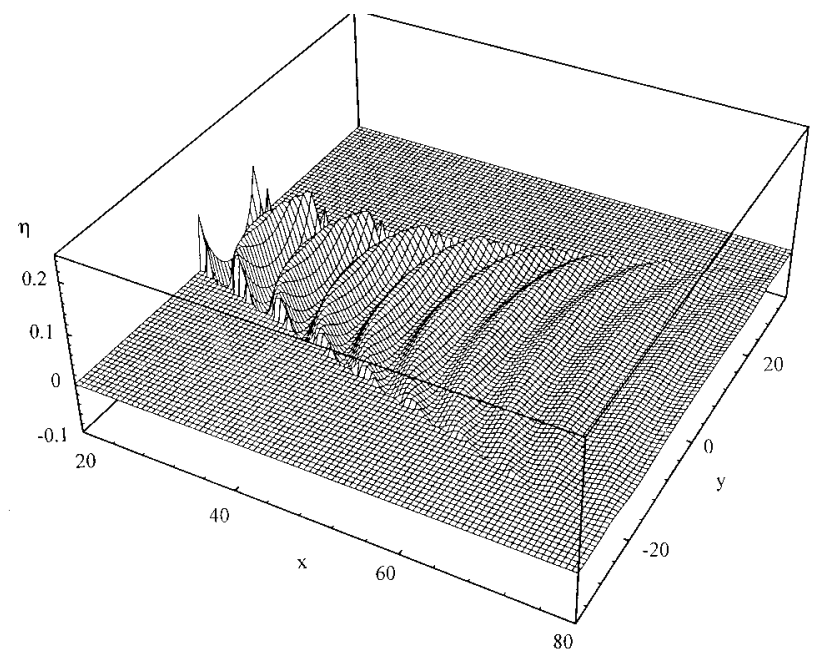

FIG. 3. The free-surface profile generated by an Oseenlet of strength $F$ $=\frac{1}{4}$ at $\epsilon=0.001, h_{1}=10$, and $h_{2}=1$.

the no-slip boundary. We can also see that at small depth the wave is much reduced and at the hypothetical position $h_{1}=1$, meaning that the Oseenlet actually touches the horizontal boundary, the entire movement is debarred, and thus no wave is expected.

In Fig. 5, the maximum wave amplitude is plotted against the submergence depth $h_{2}$ with the relative submergence depth $h_{2} / h_{1}=0.5$ at $\epsilon=0.001$. We see that the wave amplitude first rises to a maximum value and then drops to zero as the water depth increases. From the previous graphs, we know that the wave amplitude increases with water depth due to the diminishing effect of the no-slip boundary. However, at the same time, as $h_{2} / h_{1}$ is kept constant, the submergence of the Oseenlet also increases, which reduces the wave amplitude like $\exp \left[-z_{0} / \epsilon\right]$.

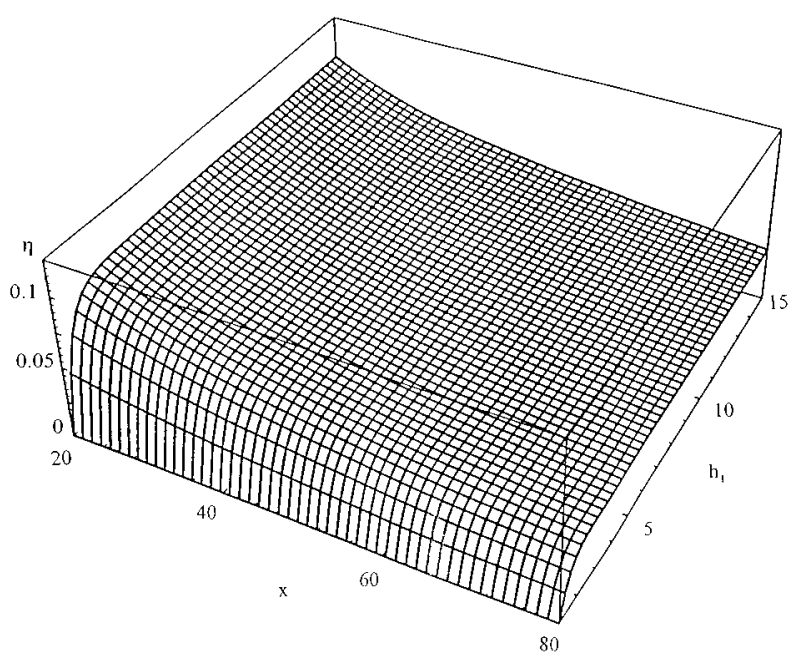

FIG. 4. The maximum wave amplitude along the line of motion of an Oseenlet versus water depth $h_{1}$ at $\epsilon=0.001$ and $h_{2}=1$.

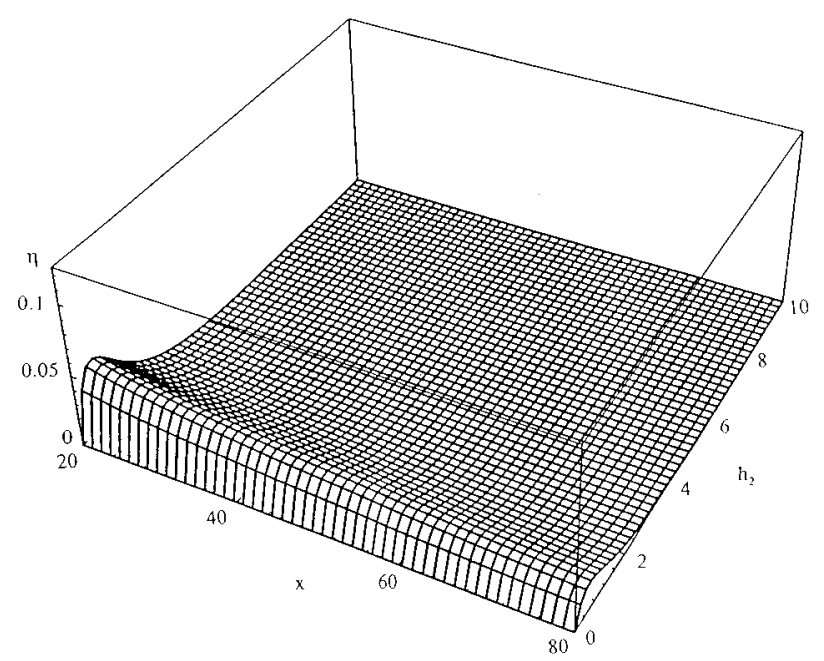

FIG. 5. The maximum wave amplitude along the line of motion of an Oseenlet versus the submergence depth $h_{2}$ at $\epsilon=0.001$ and $h_{2} / h_{1}=0.5$.

\section{CONCLUSIONS}

The wave amplitude due to an Oseenlet moving in water of finite depth is derived when the singularity is located far from the bottom and not very near the free surface. The wave amplitude can be expressed as the wave generated by the Oseenlet minus that of its mirror image plus the contribution due to a planar distribution of vertical Oseen doublets. The dominant terms of the wave amplitude, however, are those of the Oseenlet and its mirror image only. The presence of the no-slip boundary is found to reduce the wave amplitude because of the reduced velocity of the system.

\section{ACKNOWLEDGMENT}

This work was sponsored by the Committee on Research and Conference Grants, The University of Hong Kong, under Grant No. 335/064/0071.

${ }^{1}$ J. V. Wehausen and E. V. Laitone, "Surface waves," Handbuch der Physik 8/3 (Springer-Verlag, Berlin, 1960), pp. 311-778.

${ }^{2}$ E. Cumberbatch, "Effect of viscosity on ship waves," J. Fluid Mech. 23, 471 (1960).

${ }^{3}$ J. P. Dugan, "Viscous drag of bodies moving near a free surface," Phys. Fluids 12, 1 (1969).

${ }^{4}$ M. B. Wilson, "A Michell Oseen-flow theory for thin ships," Ph.D. thesis, California Institute of Technology, 1971.

${ }^{5}$ A. T. Chan and A. T. Chwang, "Interaction of laminar far-wake with a free surface,"' Phys. Fluids 8, 421 (1996).

${ }^{6}$ F. K. Moore, Theory of Laminar Flows (Princeton University Press, Princeton, NJ, 1964).

${ }^{7}$ J. R. Blake, "A note on the image system for a Stokeslet in a no-slip boundary,"' Proc. Cambridge Philos. Soc. 70, 303 (1971).

${ }^{8}$ W. E. Olmstead, "A homogeneous solution for viscous flow around a half plane," Q. Appl. Math. 33, 165 (1975).

${ }^{9}$ W. E. Olmstead and A. K. Gautesen, "Integral representations and the Oseen flow problem," Mechanics Today (Pergamon, New York, 1976), Vol. 3, pp. 125-189.

${ }^{10}$ J. R. Blake and A. T. Chwang, "Fundamental singularities of viscous flow Part I: The image systems in the vicinity of a stationary no-slip boundary,"' J. Eng. Math. 8, 23 (1974). 